\title{
Mechanical load assisted dissolution response of biomedical cobalt-chromium and titanium metallic alloys: influence of in-plane stress and chemical environment
}

Ryu, Jae-Joong, University of Texas, Tyler, United States; Shrotriya, Pranav, lowa State University, United States

\begin{abstract}
Mechanical load-assisted dissolution is identified as one of the key mechanisms governing material removal in fretting and crevice corrosion of biomedical implants. In the current study, material removal on a stressed surface of cobalt-chromium-molybdenum (CoCrMo) and titanium (Ti64) alloys subjected to single asperity contact is investigated to identify the influence of contact loads and in-plane stress state on surface damage mechanisms. The experiments were conducted in three different aqueous environments-phosphate-buffered saline (PBS) at $\mathrm{pH}$ 7.4, $\mathrm{PBS}$ at $\mathrm{pH} 4.1$, and high chloride solutions at $\mathrm{pH}$ 2.0. The tip of an atomic force microscope is used as a well-characterized "asperity" to apply controlled contact forces and mechanically stimulate the loaded specimen surface in different aqueous environments from passivating to corroding. The volume of the material removed is measured to determine the influence of contact loads, in-plane stresses, and the environment on the material dissolution rate. Experimental results indicate that surface damage is initiated at all the contact loads studied and as expected in a wear situation, removal rate increases with increase in contact loads. For both alloys, removal rates display a complex dependence on residual stresses and the environment. In a passivating environment, the material removal rate is linearly dependent on the stress state such that surface damage is accelerated under compressive stresses and suppressed under tensile stresses. In a corrosive environment, the dissolution rate demonstrates a quadratic dependence on stress, with both compressive and tensile stresses accelerating material dissolution. The material removal rates are found to be consistently larger for CoCrMo in comparison to Ti64 surfaces under the same mechanical and electrochemical stimuli, despite the higher hardness of CoCrMo surfaces. A surface damage mechanism based on stress-assisted dissolution is proposed to elucidate the experimental observations.
\end{abstract}

